\title{
Evaluation of vascular space involvement in endometrial adenocarcinomas: laparoscopic vs abdominal hysterectomies
}

\author{
Ann K Folkins ${ }^{1, *, \dagger}$, Nicole S Nevadunsky ${ }^{2, *}$, A Saleemuddin ${ }^{1}$, Elke A Jarboe ${ }^{1}$, \\ Michael G Muto ${ }^{2}$, Colleen M Feltmate ${ }^{2}$, Chris P Crum ${ }^{1}$ and Michelle S Hirsch ${ }^{1}$ \\ ${ }^{1}$ Division of Women's and Perinatal Pathology, Department of Pathology, Brigham and Women's Hospital, \\ Boston, MA, USA and ${ }^{2}$ Division of Gynecologic Oncology, Department of Obstetrics and Gynecology, Brigham \\ and Women's Hospital, Boston, MA, USA
}

\begin{abstract}
Recent reports have described 'vascular pseudoinvasion' in total laparoscopic hysterectomies with endometrial carcinoma. To better understand this phenomenon, we compared pathologic findings in these laparoscopic and total abdominal hysterectomies performed for uterine endometrioid adenocarcinoma. Reports from 58 robotically assisted laparoscopic and 39 abdominal hysterectomies with grade 1 or 2 endometrioid endometrial adenocarcinomas were reviewed for stage, depth of invasion, vascular space involvement, uterine weight, and lymph node metastases. In addition, attention was given to possible procedural artifacts, including vertical endomyometrial clefts, and inflammatory debris, benign endometrial glands, and disaggregated tumor cells in vascular spaces. All foci with vascular involvement were reviewed by three gynecologic pathologists. Nine of the $58(16 \%)$ laparoscopic and 3 of the $39(7 \%)$ abdominal hysterectomies contained vascular space involvement based on the original pathology reports $(P$-value $=\mathbf{0 . 0 8 3 3})$. No one histologic feature consistently distinguished laparoscopic from abdominal cases on blind review of the available cases. Disaggregated intravascular tumor cells were significantly associated with reported vascular involvement in both procedures $(P$-values $<0.001$ and 0.016$)$, most of which were corroborated on review. Laparoscopic procedures tend to have a higher index of vascular involvement, which is associated with lower stage, fewer lymph node metastases, and less myometrial invasion; however, pathologists cannot consistently determine the procedure on histologic findings alone. Moreover, there is significant inter-observer variability in distinguishing true from artifactual vascular space involvement, even among pathologists at the same institution. The clinical significance of apparent true vascular space involvement seen adjacent to artifacts is unclear, as is the impact of laparoscopic hysterectomy on recurrence risk.
\end{abstract}

Modern Pathology (2010) 23, 1073-1079; doi:10.1038/modpathol.2010.91; published online 14 May 2010

Keywords: laparoscopic hysterectomy; endometrial cancer; vascular space

Total laparoscopic hysterectomy is increasingly used as a safe and effective alternative to total abdominal hysterectomy for endometrial carcinomas, and has been shown to be associated with shorter

Correspondence: Dr MS Hirsch, MD, PhD, Department of Pathology, Brigham and Women's Hospital, Amory 3-127, 75 Francis Street, Boston, MA 02115, USA.

E-mail: mhirsch1@partners.org

*These two co-first authors contributed equally to this work.

"Presented at the March 2009 USCAP meeting in Boston, MA, USA.

Received 17 October 2009; revised 12 March 2010; accepted 15 March 2010; published online 14 May 2010 hospital stays and faster recovery from surgery. ${ }^{1-3}$ In most institutions, laparoscopic removal of the uterus requires the use of a balloon tip intrauterine manipulator, which is inflated with saline throughout the procedure. There has been controversy in the past years as to whether laparoscopic hysterectomies are associated with higher rates of positive peritoneal washings, presumably related to the increased intrauterine pressure from the manipulator causing tumor cells to be propelled through the fallopian tubes into the peritoneal cavity. ${ }^{4-6}$ As a result, many surgeons now ligate or clamp the tubes and/or obtain peritoneal washings before inserting the manipulator. 
Recently, two studies have addressed the possibility that balloon tip intrauterine manipulators in total laparoscopic hysterectomies for endometrial cancer are also associated with artifactual displacement of tumor cells into vascular spaces (referred to as 'vascular pseudoinvasion'). Thirty-seven laparoscopic hysterectomies for benign (30) and neoplastic (7) conditions, including six grade 1 endometrioid cancers and one atypical complex hyperplasia, were originally examined. ${ }^{7}$ The authors found tumor in blood vessels in $71 \%$ of the malignant total laparoscopic hysterectomies and benign glands and stroma in vascular spaces of $13 \%$ of benign cases. They concluded that total laparoscopic hysterectomy is associated with high rates of vascular pseudoinvasion, which is presumably caused by the increased intrauterine pressure created by the intrauterine manipulator.

The second report compared 21 total laparoscopic hysterectomies with 28 total abdominal hysterectomies performed for low-risk endometrial carcinomas and found vascular space involvement in 33\% of the total laparoscopic hysterectomies and in none of the total abdominal hysterectomies. ${ }^{8}$ In the latter they noted that the tumor cells were mostly in large vessels in the outer aspect of the myometrium but not in small vascular spaces. Sometimes, the tumor cells were associated with stroma, and the vascular space involvement was only seen in cases with exophytic tumors. Although these authors agreed with the previous study that this finding was a form of vascular pseudoinvasion, they believed that it was the result of postoperative specimen handling by the pathologist, with tumor cells being displaced during sectioning. In this scenario, the intrauterine manipulator presumably disaggregated the tumor cells, making them more prone to artificial displacement into vascular spaces during the gross pathologic examination. The authors hypothesized that if intravascular spread was due to increased intrauterine pressure alone, the tumor cells would be seen in both small and large vessels throughout the myometrium.

Resolving the cause of vascular space involvement in endometrial carcinoma, real or otherwise, is critical, in as much as the reporting of vascular space involvement can result in subsequent lymph node dissection, radiation therapy, and the impression by both surgeon and patient that the patient is at risk for recurrences. The objective of our study was to (1) assess whether vascular space involvement is more commonly reported in total laparoscopic hysterectomy than total abdominal hysterectomy performed for endometrial cancer; (2) determine whether on blind review, certain artifacts, including pseudovascular space invasion previously associated with total laparoscopic hysterectomy are specific for this procedure; and (3) determine the degree to which such artifacts might be associated with reported (and presumed to be true) vascular space involvement.

\section{Materials and methods}

This study was approved by the institutional review board at Brigham and Women's Hospital. Consecutive cases of total laparoscopic hysterectomy with robotic assistance $(N=58)$ from August 2006 through January 2009 and total abdominal hysterectomy $(N=39)$ from August 2003 to June 2005 (a period preceding minimally invasive surgery at this institution) with a diagnosis of grade 1 or grade 2 endometrial adenocarcinoma, endometrioid type, were identified at the Brigham and Women's Hospital. The reports and clinical information were used to generate a database including weight of uterus, age of patient, depth of myometrial invasion, stage, lymph node status, vascular space involvement, pelvic washing status, and possible recurrence. Slides were available for review in 55 of the total laparoscopic hysterectomy cases and 31 of the total abdominal hysterectomy cases. $P$-values were generated for vascular space involvement and uterine weight between total abdominal hysterectomy and total laparoscopic hysterectomy cases using Student's $t$-test.

Two pathologists (AKF and CPC) independently examined the slides blindly and scored them for the presence of the following four variables: (1) vertical clefts in the endomyometrium, (2) inflammatory debris in vascular spaces, and (3) benign endometrial glands in vascular spaces and disaggregated tumor cells in vascular spaces (considered artifacts by the reviewers) (Figure 1). The reviewers also noted whether they believed there was biologically significant or 'true' vascular space involvement by tumor. Criteria for true vascular space involvement were adapted from the literature, and included (1) clusters of tumors cells demonstrating a change in morphology, usually with the acquisition of pale or eosinophilic cytoplasm, (2) smooth borders, and (3) shaped to conform to the vascular space (Table 1). Perivascular lymphocytic infiltrates were considered a supportive finding.

Sensitivities and specificities were calculated for each of the variables examined in relationship to total laparoscopic hysterectomy and also to the presence of vascular space involvement within the original pathology report. $\chi^{2}$ analysis was used to generate $P$-values for all sensitivities and specificities. $\kappa$-values with confidence intervals were also calculated for each variable. All cases in which either the original report or one of the two reviewers identified 'true' vascular space involvement were re-reviewed by a panel of three gynecologic pathologists (AKF, CPC, and MSH) for a consensus (or majority) diagnosis (Figure 2).

\section{Results}

\section{Hysterectomy Findings}

There was no significant difference $(P$-value $=0.5355)$ in the uterine weight between the laparoscopic 

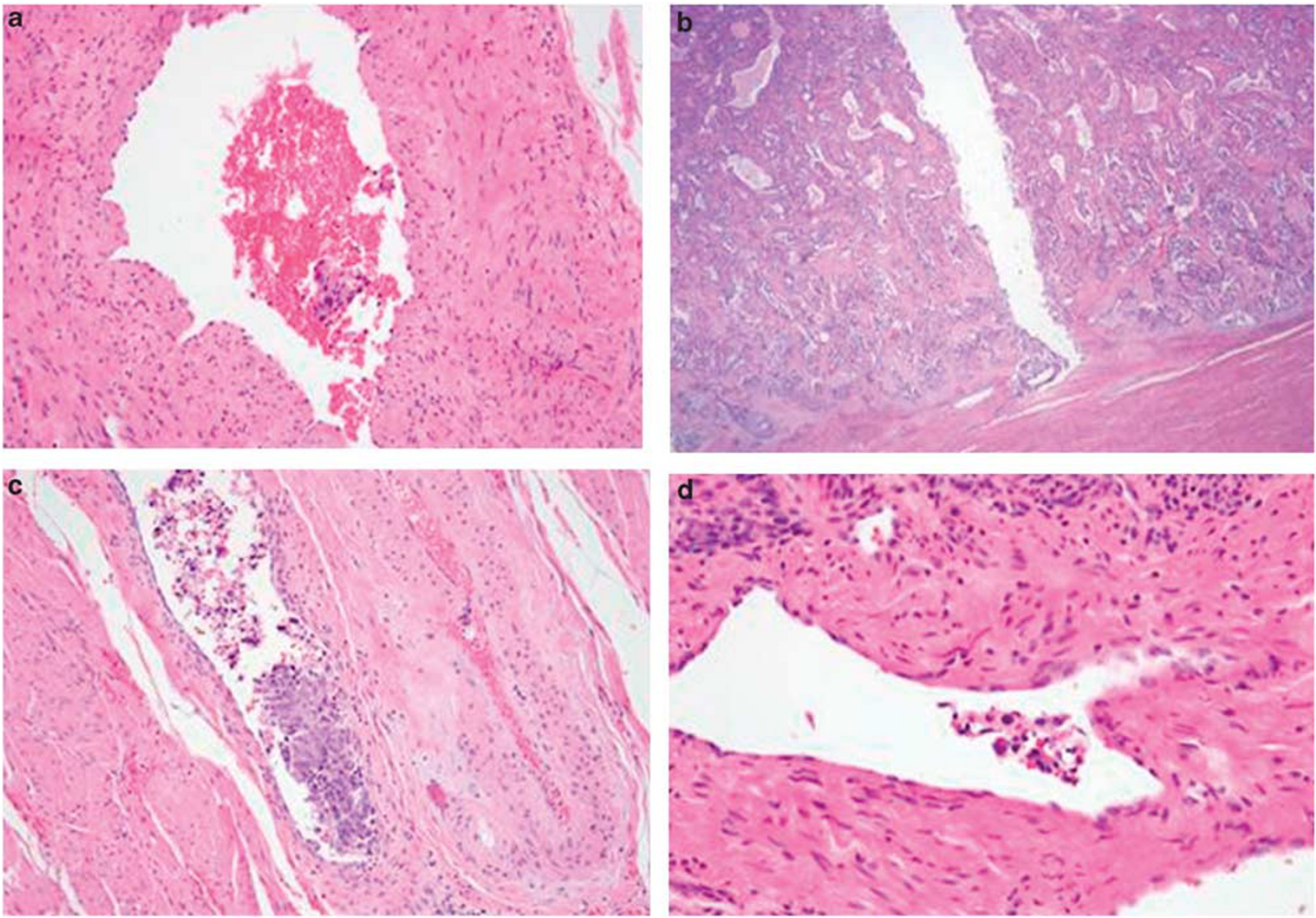

Figure 1 Artifacts cataloged in the study include (a) benign endometrial tissue; (b) vertical clefts; (c) disaggregated tumor cells; and (d) inflammatory debris.

Table 1 Criteria for true and artifactual lymphovascular involvement

\begin{tabular}{ll}
$\begin{array}{l}\text { True lymphovascular space } \\
\text { involvement }\end{array}$ & $\begin{array}{l}\text { Artifactual lymphovascular } \\
\text { space involvement }\end{array}$ \\
\hline $\begin{array}{l}\text { Smooth bordered cohesive } \\
\text { clusters of tumor cells }\end{array}$ & $\begin{array}{l}\text { Disaggregated tumor cells, often } \\
\text { intermixed with inflammation }\end{array}$ \\
$\begin{array}{l}\text { Conform to the contours of } \\
\text { the lymphovascular space }\end{array}$ & $\begin{array}{l}\text { Floating in the lymphovascular } \\
\text { space }\end{array}$ \\
$\begin{array}{l}\text { Often a change in morphology } \\
\text { with more eosinophilic }\end{array}$ & $\begin{array}{l}\text { Similar morphology to intact } \\
\text { tumor, sometimes as intact } \\
\text { cytoplasm }\end{array}$ \\
$\begin{array}{l}\text { fymphatic space adjacent } \\
\text { to larger vessels }\end{array}$ & $\begin{array}{l}\text { Spaces are immediately } \\
\text { adjacent to invasive tumor with } \\
\text { retraction artifact }\end{array}$ \\
\hline
\end{tabular}

hysterectomies with vascular space involvement (mean $148 \mathrm{~g}$ ) and abdominal hysterectomies with vascular space involvement (mean $118 \mathrm{~g}$ ) cases. Laparoscopic hysterectomy and abdominal hysterectomy cases showed similar low clinical stage (ie, only one fourth of the cases were FIGO stage $1 \mathrm{C}$ or greater). In our series, according to the final pathology reports, the rate of vascular space involvement did not significantly differ between total laparoscopic hysterectomy and total abdominal hysterectomy cases although there was a trend towards a higher rate with the laparoscopic procedure. Nine of $58(16 \%)$ laparoscopic hysterectomies and three of $39(7 \%)$ abdominal hysterectomies reported vascular space involvement $(P=0.0833)$. The nine laparoscopic hysterectomies with vascular space involvement ranged from FIGO stage $1 \mathrm{~A}$ to 3C $(44 \% \geq$ stage $1 \mathrm{C})$ with $67 \%$ (six cases) demonstrating $<50 \%$ myometrial invasion. The three total abdominal hysterectomies with vascular space involvement ranged from FIGO stage $1 \mathrm{C}$ to $3 \mathrm{C}$ (none were stage $1 \mathrm{~A}$ or $1 \mathrm{~B}$ ) with only $34 \%$ (one case) demonstrating $<50 \%$ myometrial invasion. Lymph nodes were sampled in five of the nine total laparoscopic hysterectomies + vascular space involvement, and only one case had metastatic disease $(20 \%)$. In contrast, lymph nodes were sampled in all three total abdominal hysterectomies + vascular space involvement, and two of the threes cases showed positive lymph nodes (66\%). Thus, overall there was a trend toward total laparoscopic hysterectomy + vascular space involvement being lower stage, with shallower myometrial invasion, and fewer positive lymph nodes. The sample size was small and not large enough for statistical analysis. 

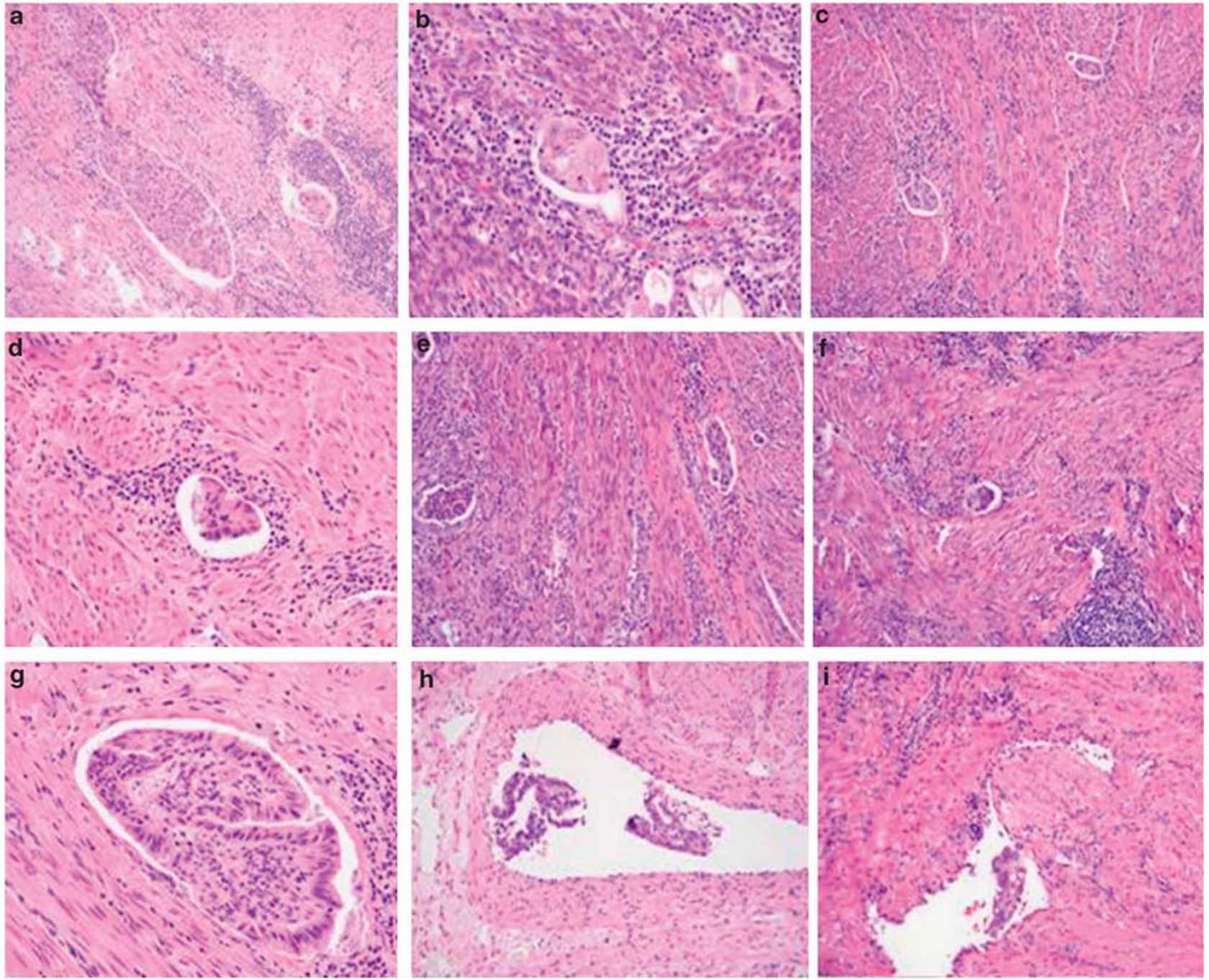

Figure 2 Microscopic fields reflecting a consensus diagnosis of vascular space involvement (a-c), suspicious for vascular space involvement $(\mathbf{d}-\mathbf{f})$, and presumed artifact $(\mathbf{g}-\mathbf{i})$.

\section{Histologic Artifacts}

Cases were evaluated for four specific histologic artifacts (shown in Figure 1); however, none could reliably or reproducibly distinguish total laparoscopic hysterectomy from total abdominal hysterectomy, despite the fact that one of the three pathologists in a pilot review was able to identify laparoscopic hysterectomy + vascular space involvement and abdominal hysterectomy + vascular space involvement in 9 out of 10 and 7 out of 10 cases, respectively. This was based on the presence of endomyometrial vertical clefts and inflammatory debris in vascular spaces. Unfortunately, such distinction could not be confirmed in a larger analysis of all the available cases. The sensitivities and specificities of the variables designated as 'artifactual' in total laparoscopic hysterectomy cases are shown in Tables 2 and 3 , and illustrate the agreement between at least two observers on these parameters. The presence of vertical clefts in the endomyometrium was specific
(94\%) but not sensitive (15\%) for total laparoscopic hysterectomy for reviewer number 1; however, it showed poor inter-observer reproducibility $(\kappa$-value $=0.073)$ and poor specificity and sensitivity for total laparoscopic hysterectomy by reviewer number 2. The presence of inflammatory vascular debris was relatively specific for total laparoscopic hysterectomy cases, but not very sensitive, and the two reviewers showed $79 \%$ agreement in identifying these findings, respectively $(\kappa$-value $=0.41)$. Disaggregated tumor cells in vascular spaces were neither sensitive nor specific for total laparoscopic hysterectomy, and the two observers showed good agreement on this finding. Although the presence of benign endometrial glands in vascular spaces was very specific for total laparoscopic hysterectomy (100 and $94 \%$ by the reviewers), it was again not sensitive, and the two reviewers did not agree as well on which cases showed this finding. The poor $\kappa$-value for this variable reflects the fact that the two reviewers identified different cases with this finding. 
Table 2 Reviewer assessment of parameters associated with hysterectomy

\begin{tabular}{|c|c|c|c|c|c|c|c|c|}
\hline Rev & Variable & TLH+ & $T A H(+)$ & $T L H(-)$ & $T A H(-)$ & $\begin{array}{l}\text { Sensitivity } \\
\text { for TLH }\end{array}$ & $\begin{array}{c}\text { Specificity } \\
\text { for TLH }\end{array}$ & $\mathrm{P}$ \\
\hline \multirow[t]{4}{*}{1} & Vertical clefts in the myometrium & 8 & 2 & 47 & 29 & 14.55 & 93.55 & 0.32 \\
\hline & Inflammatory debris in vascular spaces & 13 & 6 & 42 & 25 & 23.64 & 80.65 & 0.79 \\
\hline & Benign glands in vascular spaces & 3 & 0 & 52 & 31 & 5.45 & 100.00 & 0.55 \\
\hline & Disaggregated tumor cells in lymphovascular spaces & 14 & 9 & 41 & 22 & 25.45 & 70.97 & 0.8 \\
\hline \multirow[t]{4}{*}{2} & Vertical clefts in the myometrium & 30 & 16 & 25 & 15 & 54.55 & 48.39 & 0.83 \\
\hline & Inflammatory debris in vascular spaces & 17 & 4 & 38 & 27 & 30.91 & 87.10 & 0.072 \\
\hline & Benign glands in vascular spaces & 2 & 2 & 53 & 29 & 3.64 & 93.55 & 0.62 \\
\hline & Disaggregated tumor cells in lymphovascular spaces & 16 & 10 & 39 & 21 & 29.09 & 67.74 & 0.81 \\
\hline
\end{tabular}

Table 3 Agreement among two observers for parameters associated with laparoscopic vaginal hysterectomy

\begin{tabular}{lccc}
\hline & Pct agreement & Kappa value & Confidence interval \\
\hline Vertical clefts in the myometrium & 51.16 & 0.073 & -0.053 to 0.198 \\
Inflammatory debris in lymphovascular spaces & 79.07 & 0.41 & 0.189 to 0.639 \\
Benign glands in lymphovascular spaces & 91.86 & -0.042 & -0.074 to -0.01 \\
Disaggregated tumor cells in lymphovascular spaces & 82.56 & 0.57 & 0.38 to 0.76 \\
\hline
\end{tabular}

Table 4 Association of parameters associated with laparoscopic vaginal hysterectomy with the diagnosis of lymphovascular invasion

\begin{tabular}{|c|c|c|c|c|c|c|c|c|}
\hline Rev & Variable & $\begin{array}{l}\text { LVI+ with } \\
\text { variable }\end{array}$ & $\begin{array}{l}\text { LVI(-) with } \\
\text { variable }\end{array}$ & $\begin{array}{l}\text { LVI+ without } \\
\text { variable }\end{array}$ & $\begin{array}{l}\text { LVI }(-) \text { without } \\
\text { variable }\end{array}$ & $\begin{array}{l}\text { Sensitivity } \\
\quad \text { for LVI }\end{array}$ & $\begin{array}{l}\text { Specificity } \\
\quad \text { for LVI }\end{array}$ & $\mathrm{P}$ \\
\hline \multirow[t]{4}{*}{1} & Vertical clefts in the endomyometrium & 2 & 8 & 8 & 68 & 20.00 & 89.47 & 0.61 \\
\hline & Inflammatory debris in vascular spaces & 7 & 12 & 3 & 64 & 70.00 & 84.21 & 0.002 \\
\hline & Benign glands in lymphovascular spaces & 1 & 2 & 9 & 74 & 10.00 & 97.37 & 0.34 \\
\hline & $\begin{array}{l}\text { Disaggregated tumor cells in } \\
\text { lymphovascular spaces }\end{array}$ & 8 & 15 & 2 & 61 & 80.00 & 80.26 & 0.001 \\
\hline \multirow[t]{4}{*}{2} & Vertical clefts in the endomyometrium & 5 & 41 & 5 & 35 & 50.00 & 56.05 & 1 \\
\hline & Inflammatory debris in vascular spaces & 5 & 16 & 5 & 60 & 50.00 & 78.95 & 0.13 \\
\hline & Benign glands in lymphovascular spaces & 0 & 4 & 10 & 72 & 00.00 & 94.74 & 1 \\
\hline & $\begin{array}{l}\text { Disaggregated tumor cells in } \\
\text { lymphovascular spaces }\end{array}$ & 7 & 19 & 3 & 57 & 70.00 & 75.00 & 0.016 \\
\hline
\end{tabular}

\section{Histologic Artifacts and Vascular Space Involvement}

When the presumed artifacts were correlated with the presence of vascular space involvement reported in the original pathology report (Table 4), there was a significant association between the finding of vascular space involvement and disaggregated tumor cells within vascular spaces for both reviewers $(P$-value $<0.001 ; \quad P$-value $=0.016)$. For reviewer number 1, inflammatory debris in vascular spaces was also significantly associated with vascular space involvement $(P$-value $=0.002)$. Vertical clefts and benign endometrial glands in vascular spaces showed no significant association with vascular space involvement for either reviewer.

\section{True vs Artifactual Vascular Space Involvement}

The results of the reviewers' agreement with the original diagnosis of vascular space involvement in the pathology report and with each other are shown in Table 5. Of the 10 total cases with reported vascular space involvement (seven total laparoscopic hysterectomy and three total abdominal hysterectomy), reviewer 1 identified eight as true vascular space involvement, and reviewer 2 identified seven. In addition, the reviewers agreed on the presence of vascular space involvement in 6 of the 10 cases $(60 \%)$. In one case, neither reviewer identified the originally reported vascular space involvement; in the remaining three cases, the reviewers disagreed on whether the vascular space involvement was real or artifactual. In addition, reviewer 1 found three more cases of vascular space involvement that were not originally diagnosed, and reviewer 2 found four more cases of vascular space involvement that were not originally diagnosed; two of these cases were identified by both reviewers independently, leaving five additional cases with vascular space involvement.

The panel of three pathologists collectively and blindly reviewed all 15 cases (10 identified from the 
Table 5 Initial and final assessments of lymphovascular invasion among observers

\begin{tabular}{lllll}
\hline $\begin{array}{l}\text { TLH or } \\
\text { TAH }\end{array}$ & $\begin{array}{l}\text { Original report } \\
\text { with LVI }\end{array}$ & $\begin{array}{l}\text { Rev } 1 \\
\text { LVI }\end{array}$ & $\begin{array}{l}\text { Rev } 2 \\
\text { LVI }\end{array}$ & $\begin{array}{l}\text { Consensus } \\
\text { LVI }\end{array}$ \\
\hline TAH & Yes & Yes & Yes & Yes \\
TAH & Yes & Yes & Yes & Suspicious \\
TAH & Yes & No & Yes & Yes \\
TLH & Yes & No & No & Suspicious \\
TLH & Yes & Yes & Yes & Yes \\
TLH & Yes & Yes & Yes & Yes \\
TLH & Yes & Yes & Yes & Yes \\
TLH & Yes & Yes & Yes & Yes \\
TLH & Yes & Yes & No & No \\
TLH & Yes & Yes & No & No \\
TAH & No & No & Yes & No \\
TLH & No & Yes & No & No \\
TLH & No & No & Yes & No \\
TLH & No & Yes & Yes & Yes \\
TLH & No & Yes & Yes & Suspicious \\
& & & & \\
\hline
\end{tabular}

original pathology report plus 5 identified during this study) of possible vascular space involvement, and concluded that (1) 2 of the 10 cases $(20 \%)$ originally called vascular space involvement in pathology reports were in fact artifactual and that (2) only one of five cases $(20 \%)$ identified by one or both of the reviewers during this study as vascular space involvement was definitely true vascular space involvement, and that the other four cases $(80 \%)$ were artifactual displacement of tumor into vascular spaces. To summarize, true vascular space involvement is composed of smooth bordered and cohesive clusters of tumor cells that conform to the contours of the vascular space. The cells often show a change in morphology with more eosinophilic cytoplasm and are often present in lymphatic spaces adjacent to larger vessels. In contrast, artifactual vascular space involvement is often composed of disaggregated tumor cells that are often intermixed with inflammation and are floating freely or in fragments in the vascular space. The cells often show similar morphology to the main tumor mass and are often present in spaces immediately adjacent to invasive tumor with retraction artifact.

\section{Discussion}

In contrast to the two previously published studies, which addressed vascular space involvement by endometrial carcinoma in total laparoscopic hysterectomies, ${ }^{7,8}$ this study did not find a significant difference in the rate of vascular space involvement between total laparoscopic hysterectomy and total abdominal hysterectomy. Nevertheless, there was a trend towards a higher frequency of vascular space involvement in the total laparoscopic hysterectomy group and the lack of a strong correlation can be related to a number of issues. First, there may be institutional differences in the amount of saline used to insufflate the intrauterine manipulator for total laparoscopic hysterectomy. At our institution, $5 \mathrm{ml}$ of saline is the standard, but can be up to $8 \mathrm{ml}$ if the intrauterine cavity is large. Second, the weight of the uterus might affect the rate of reported vascular space involvement because the uterus will be compressed when removed vaginally during the total laparoscopic hysterectomy. However, in our study, there was no significant difference in uterine weight between total laparoscopic hysterectomy + vascular space involvement and total abdominal hysterectomy + vascular space involvement cases arguing against this hypothesis. The third and probably most likely reason is that pathologists at different institutions, and even within the same institution, may use different thresholds for defining vascular space involvement. In this study, only 8 of 10 cases originally reported as vascular space involvement during the diagnostic procedure could be confirmed as true vascular space involvement by a consensus panel of three pathologists. Moreover, there was some inter-observer disagreement over what constituted real vascular space involvement in blinded reviews.

Prior studies have suggested that disaggregated tumor cells in vascular spaces (so called 'vascular pseudoinvasion') are seen more frequently in total laparoscopic hysterectomy cases. However, our blinded re-review of total laparoscopic hysterectomy + vascular space involvement and total abdominal hysterectomy + vascular space involvement cases did not reveal a significant correlation between disaggregated tumor cells in vascular spaces and procedure type, implying that this type of artifact can be seen in both total laparoscopic hysterectomy and total abdominal hysterectomy cases. On the other hand, this study did show that there is a correlation between the presence of disaggregated tumor cells in vascular spaces and the presence of 'true' vascular space involvement as reported by the original pathologist and summarized in Table 1 . The difficulties in reproducing these categories on review is illustrated in this report. Ten cases were initially reported as vascular space involvement on the original pathology report. Five additional cases were identified as containing vascular space involvement by at least one of the two pathologists participating in the review of cases. When the three pathologists convened to resolve these differences in interpretation, 2 the 10 original vascular space involvement diagnoses were rejected and only one of the five additional interpretations of vascular space involvement was confirmed by consensus opinion. This illustrates that there can be significant inter-observer variability, even among pathologists within the same department. This underscores the difficulties of assessing vascular space invasion and has implications for clinical management.

In summary, this study has shown that vascular space involvement tends to occur more frequently following laparoscopic procedures and in such cases, there is a trend towards a lower stage relative to 
abdominal procedures. However, despite this, space involvement deemed as both artifact and real can co-exist in the same case and the origin (real vs artifact) cannot be consistently sorted out by a single reviewer. The lower reported rates of lymph node involvement in these cases suggest that in some instances, artifactual space involvement may be indistinguishable from real vascular invasion. Resolving this will require long-term follow-up data to determine whether the subset of cases interpreted as both real and artifactual space involvement are at greater risk for local or distant recurrence. Pending such data, we support grossing procedures that theoretically minimize the possibility of artifact, as outlined earlier. ${ }^{8}$

\section{Disclosure/conflict of interest}

The authors declare no conflict of interest.

\section{References}

1 Ghezzi F, Cromi A, Bergamini V, et al. Laparoscopicassisted vaginal hysterectomy versus total laparoscopic hysterectomy for the management of endometrial cancer: a randomized clinical trial. J Minim Invasive Gynecol 2006;13:114-120.
2 Kalogiannidis I, Lambrechts S, Amant F, et al. Laparoscopy-assisted vaginal hysterectomy compared with abdominal hysterectomy in clinical stage I endometrial cancer: safety, recurrence, and long-term outcome. Am J Obstet Gynecol 2007;196:248.

3 Malzoni M, Tinelli R, Cosentino F, et al. Total laparoscopic hysterectomy versus abdominal hysterectomy with lymphadenectomy for early-stage endometrial cancer: a prospective randomized study. Gynecol Oncol 2009;112:126-133.

4 Eltabbakh GH, Mount SL. Laparoscopic surgery does not increase the positive peritoneal cytology among women with endometrial carcinoma. Gynecol Oncol 2006;100:361-364.

5 Lim S, Kim HS, Lee KB, et al. Does the use of a uterine manipulator with an intrauterine balloon in total laparoscopic hysterectomy facilitate tumor cell spillage into the peritoneal cavity in patients with endometrial cancer? Int J Gynecol Cancer 2008;18:1145-1149.

6 Sonoda Y, Zerbe M, Smith A, et al. High incidence of positive peritoneal cytology in low-risk endometrial cancer treated by laparoscopically assisted vaginal hysterectomy. Gynecol Oncol 2001;80:378-382.

7 Logani S, Herdman AV, Little JV, et al. Vascular 'pseudo invasion' in laparoscopic hysterectomy specimens: a diagnostic pitfall. Am J Surg Pathol 2008;32: $560-565$.

8 Kitahara S, Walsh C, Frumovitz M, et al. Vascular pseudoinvasion in laparoscopic hysterectomy specimens for endometrial carcinoma: a grossing artifact? Am J Surg Pathol 2009;33:298-303. 